University of Nebraska - Lincoln

DigitalCommons@University of Nebraska - Lincoln

\title{
Absence of local magnetic moments in Ru and Rh impurities and clusters on $\mathrm{Ag}(100)$ and $\mathrm{Pt}(997)$
}

\author{
Jan Honolka \\ Max-Planck-Institut für Festkörperforschung, honolka@fzu.cz \\ K. Kuhnke \\ Max-Planck-Institut für Festkörperforschung \\ L. Vitali \\ Max-Planck-Institut für Festkörperforschung \\ Axel Enders \\ University of Nebraska-Lincoln, a.enders@me.com \\ K. Kern \\ Max-Planck-Institut für Festkörperforschung, k.kern@fkf.mpg.de \\ See next page for additional authors
}

Follow this and additional works at: https://digitalcommons.unl.edu/physicsenders

Part of the Physics Commons

Honolka, Jan; Kuhnke, K.; Vitali, L.; Enders, Axel; Kern, K.; Gardonio, S.; Carbone, C.; Krishnakumar, S. R.; Bencok, P.; Stepanow, S.; and Gambardella, P., "Absence of local magnetic moments in Ru and Rh impurities and clusters on $\mathrm{Ag}(100)$ and Pt(997)" (2007). Axel Enders Publications. 8.

https://digitalcommons.unl.edu/physicsenders/8

This Article is brought to you for free and open access by the Research Papers in Physics and Astronomy at DigitalCommons@University of Nebraska - Lincoln. It has been accepted for inclusion in Axel Enders Publications by an authorized administrator of DigitalCommons@University of Nebraska - Lincoln. 


\section{Authors}

Jan Honolka, K. Kuhnke, L. Vitali, Axel Enders, K. Kern, S. Gardonio, C. Carbone, S. R. Krishnakumar, P. Bencok, S. Stepanow, and P. Gambardella 


\title{
Absence of local magnetic moments in $\mathrm{Ru}$ and $\mathrm{Rh}$ impurities and clusters on $\operatorname{Ag}(100)$ and $\operatorname{Pt}(997)$
}

\author{
J. Honolka, * K. Kuhnke, L. Vitali, A. Enders, and K. Kern \\ Max-Planck-Institut für Festkörperforschung, Heisenbergstrasse 1, 70569 Stuttgart, Germany \\ S. Gardonio and C. Carbone \\ Istituto di Struttura della Materia, Consiglio Nazionale delle Ricerche, Area Science Park, 34012 Trieste, Italy \\ S. R. Krishnakumar \\ International Centre for Theoretical Physics (ICTP), Strada Costiera 11, 34100 Trieste, Italy \\ P. Bencok \\ European Synchrotron Radiation Facility, Bôte Postale 200, 38043 Grenoble, France \\ S. Stepanow \\ Centre d'Investigacions en Nanociència i Nanotecnologia (CSIC-ICN), UAB Campus, 08193 Bellaterra, Spain \\ P. Gambardella \\ Institució Catalana de Recerca i Estudis Avançats (ICREA) and Centre d'Investigacions en Nanociència i Nanotecnologia (CSIC-ICN), \\ UAB Campus, 08193 Bellaterra, Spain
}

(Received 30 January 2007; revised manuscript received 7 September 2007; published 9 October 2007)

\begin{abstract}
The magnetism of quench-condensed $\mathrm{Ru}$ and $\mathrm{Rh}$ impurities and metal films on $\operatorname{Ag}(100)$ and $\mathrm{Pt}(997)$ has been studied using x-ray magnetic circular dichroism. In the coverage range between 0.22 and 2.0 ML, no dichroic signal was detected at the $M_{3,2}$ absorption edges of $\mathrm{Ru}$ on $\mathrm{Ag}(100)$ at a temperature of $5 \mathrm{~K}$ in the presence of an applied magnetic field. The same was found for coverages between 0.12 and $0.5 \mathrm{ML}$ of $\mathrm{Rh}$ on $\operatorname{Ag}(100)$ and $\mathrm{Pt}(997)$. It is concluded that the magnetic moments of single impurities, small clusters of various shape, and monolayers of the $4 d$ metals are below the detection limit of $0.04 \mu_{B}$ per atom. These results provide an unambiguous determination of the local magnetic moment of $\mathrm{Ru}$ and $\mathrm{Rh}$ deposited on nonmagnetic transition-metal surfaces, which are in contrast with theoretical predictions.
\end{abstract}

DOI: 10.1103/PhysRevB.76.144412

PACS number(s): 75.20.Hr, 78.20.Ls, 78.70.Dm

\section{INTRODUCTION}

The search for magnetism in $4 d$ metal elements has generated a large number of theoretical and experimental investigations, often in contradiction with each other. SternGerlach experiments have shown that Rh clusters of 10-30 atoms in molecular beams possess a magnetic moment of about $1-0.5 \mu_{B}$ per atom, which tends to vanish with increasing cluster size. ${ }^{1,2}$ This result has been generally explained by the reduced coordination of the $4 d$ atoms, which narrows the width of the electronic bands and increases the density of states at the Fermi level so as to fulfill the Stoner criterium for the appearance of ferromagnetism. Similar arguments applied to monolayer films and clusters deposited on nonmagnetic substrates have stimulated $a b$ initio theoretical efforts, which predicted $4 d$ magnetism for a broad variety of nanostructures, including clusters of different sizes and shapes, ${ }^{3-6}$ atomic chains,,${ }^{7,8}$ and mono- or bilayers. ${ }^{9-14}$ According to systematic calculations by Eriksson et al. ${ }^{9}$ and Blügel, ${ }^{12}$ overlayers of the late $4 d$ elements $\mathrm{Rh}$ and $\mathrm{Ru}$ exhibit ferromagnetism when placed on a $\operatorname{Ag}(100)$ substrate with magnetic moments of $1.0 \mu_{B}$ and $1.7 \mu_{B}$ per atom, respectively. Calculations of clusters with finite dimensions for coverages below $1 \mathrm{ML}$ predict that the $4 d$ magnetic moment significantly depends on geometry and substrate: compact clusters as well as elongated chain configurations on $\mathrm{Ag}(100)$ present finite moments varying from 0.3 to $2.0 \mu_{B}{ }^{4-8}$ In the case of isolated adatoms (impurities), both $\mathrm{Ru}$ and $\mathrm{Rh}$ are expected to be magnetic on $\operatorname{Ag}(100)$, with local moments of $2.2 \mu_{B}$ and $0.3 \mu_{B}$, respectively. ${ }^{3,4}$ In all these studies, it has been stressed that the values of the moments vary strongly with the local coordination of $4 d$ atoms. Moreover, according to theory, the intermixing of Rh and Ru layers with the $\mathrm{Ag}$ surface layers as well as the growth of imperfect films with noninteger coverage between 1 and 2 ML can strongly decrease $4 d$ magnetism, ${ }^{13,14}$ making its experimental verification rather difficult.

So far, most experiments have concentrated on Rh systems. Magneto-optical Kerr (MOKE) investigations of $1 \mathrm{ML}$ $\mathrm{Rh} / \mathrm{Ag}(100),{ }^{15}$ 0.5-5 ML Rh/Au(100), ${ }^{16}$ and 0-6 ML $\mathrm{Rh} / \mathrm{Au}(111)$ (Ref. 17) performed at temperatures down to 40,100 , and $30 \mathrm{~K}$, respectively, indeed failed to confirm the presence of ferromagnetism in Rh. Several explanations have been put forward to reconciliate the MOKE results with theoretical calculations. It was remarked that the impossibility to grow ideal $4 d$ monolayers, i.e., the formation of a diffuse $\mathrm{Rh}-\mathrm{Ag}$ interface as well as three-dimensional island growth, could induce a significant reduction of the $\mathrm{Rh}$ magnetism. ${ }^{13,14}$ Structural imperfections, strain relaxation, and intermixing are typical features of epitaxial films grown 
out of equilibrium that can scarcely be taken into account by band-structure models. Moreover, as ab initio calculations are usually carried out at $0 \mathrm{~K}$, the lack of ferromagnetism could be attributed to the limited temperature range probed by MOKE experiments, specifically if the Curie temperature is situated below 30-40 K. The existence of long-range ferromagnetic order in $4 d$ metal layers, however, is not the only prediction that can be experimentally tested. Prior to that, in fact, one should prove that $\mathrm{Rh}$ and $\mathrm{Ru}$ atoms deposited on a noble metal surface preserve part of their gas-phase local magnetic moment. MOKE performed at relatively high temperature and low magnetic fields $(\leqslant 0.2 \mathrm{~T})$ does not yield information in this respect. Early photoemission measurements showed a splitting of the Rh $4 s$ core levels for 1-3 ML $\mathrm{Rh} / \mathrm{Ag}(100)$, which was taken as an indicator for the presence of a local magnetic moment. ${ }^{18}$ In a more recent study, Beckmann and Bergmann have investigated $\mathrm{Rh}(\mathrm{Ru})$ impurities quench condensed on $\mathrm{Au}(\mathrm{Au}$ and $\mathrm{Ag}$ ) films grown on quartz supports by measuring the film magnetoresistance and anomalous Hall effect. ${ }^{19}$ By modeling the dephasing of electrons scattered from impurities, these authors concluded that $\mathrm{Ru}$ impurities on $\mathrm{Au}$ and $\mathrm{Ag}$ films possess a small but finite moment of about $0.4 \mu_{B}$ and suggested that Rh clusters have a fluctuating moment of the order of $0.1 \mu_{B}$. These interesting results outlined a nontrivial magnetic behavior of $4 d$ metal overlayers, even though the estimate of the $\mathrm{Ru}$ and $\mathrm{Rh}$ magnetization was performed in a nonlocal way, measuring the electrical properties of the supporting films. ${ }^{19,20}$

In this work, we report on element-specific x-ray magnetic circular dichroism (XMCD) measurements of the local magnetic moment of $\mathrm{Ru}$ and $\mathrm{Rh}$ adatoms and cluster ensembles deposited at $5 \mathrm{~K}$ on $\mathrm{Ag}$ and Pt surfaces. We show that no magnetic moment is detected in the coverage range between 0.12 and $2.0 \mathrm{ML}$, independent of the magnitude of externally applied static magnetic fields. These results show unambiguously that $\mathrm{Ru}$ and $\mathrm{Rh}$ in the form of impurities, small clusters, and quench-condensed films possess negligible local magnetic moments under static field conditions, in contrast to theoretical predictions. The XMCD data call either for a revision of existing $a b$ initio models or for taking into account many-body, temperature-dependent effects that can induce spin fluctuations over time scales faster than the time resolution of the present experiment.

\section{EXPERIMENT}

The measurements were carried out at beamline ID08 of the European Synchrotron Radiation Facility (ESRF) in Grenoble. Sample preparation and magnetic characterization were performed in situ under ultrahigh vacuum conditions. Single-crystal $\operatorname{Ag}(100)$ and $\operatorname{Pt}(997)$ surfaces were cleaned by repeated sputter and annealing cycles until a satisfactory low-energy electron diffraction pattern was obtained and no contaminants were detected by $\mathrm{x}$-ray absorption spectroscopy (XAS). In order to prevent surface diffusion and intermixing, the $4 d$ metals were deposited at a temperature of $T$ $=5 \mathrm{~K}$ using an e-beam evaporator directly connected to the XAS-XMCD vacuum chamber. Contamination checks prior and after the measurements were done on the O XAS absorp-
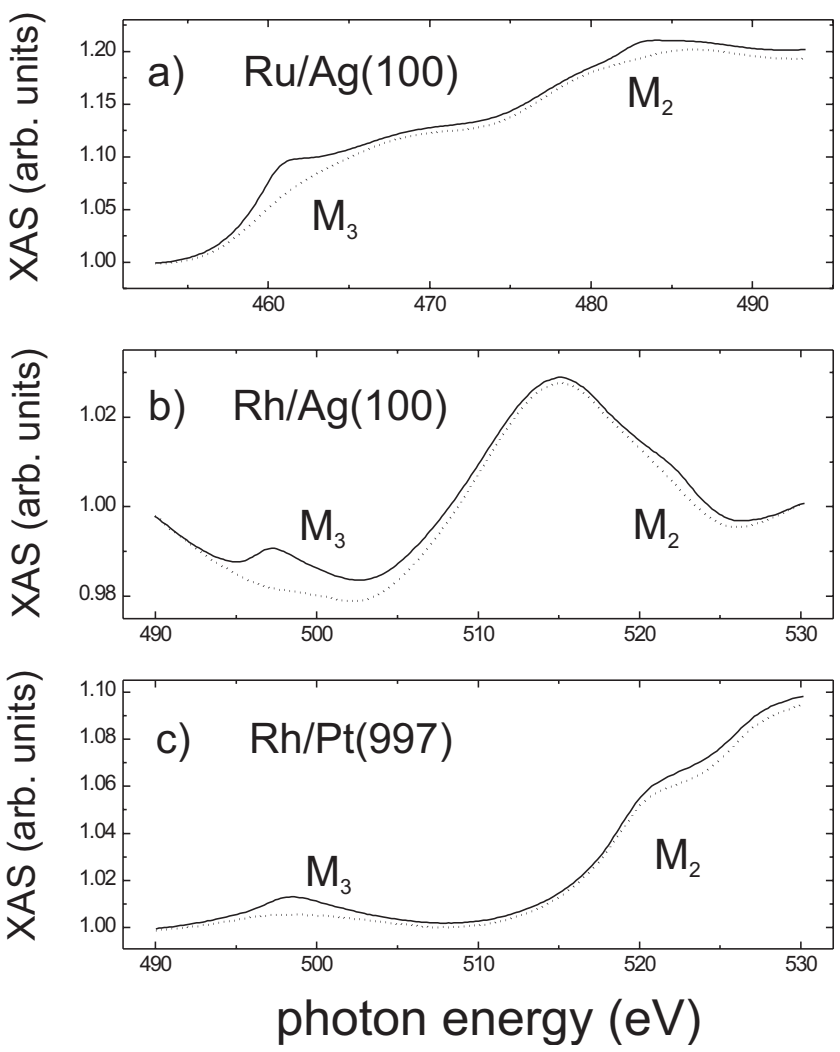

FIG. 1. $M_{3,2}$ XAS spectra of (a) Ru (0.22 ML), (b) Rh (0.09 ML) deposited on $\mathrm{Ag}(100)$, and (c) $\mathrm{Rh}$ (0.12 ML) on $\mathrm{Pt}(997)$ at $T$ $=5 \mathrm{~K}$. The spectra were normalized to unity at the $M_{3}$ preedge energies. The background XAS measured prior to deposition on pristine $\mathrm{Ag}$ and $\mathrm{Pt}$ substrates is represented by dotted lines.

tion line to exclude possible contamination from oxygen, carbon monoxide, and water. Room temperature scanning tunneling microscopy (STM) was used, prior to the XMCD measurements, to further verify the quality of the substrates and, after the XMCD measurements, to estimate the total $4 d$ overlayer coverage. Intermediate coverages were determined by linear extrapolation of the evaporation time at constant evaporation rate, which was monitored in real time by the current of ionized atoms reaching the sample. XAS spectra were taken in the region of the $M_{3,2}$ lines of $\mathrm{Rh}(\mathrm{Ru})$, situated at energies of $497 \mathrm{eV}(461 \mathrm{eV})$ and $522 \mathrm{eV}(483 \mathrm{eV})$. As shown in Fig. 1 at low coverages, the $M_{3,2}$ lines are superimposed by a rather large background signal from the substrates. The structures in the background intensity originate from the $\operatorname{Ag} M_{5,4}$ thresholds situated at lower energy, while for Pt the increase of background intensity around $525 \mathrm{eV}$ stems from the $N_{3}$ absorption edge. The XAS intensity was measured by recording the total photoelectron current by means of an electrometer as a function of the x-ray energy and positive $\left(\sigma^{+}\right)$or negative $\left(\sigma^{-}\right)$x-ray circular polarization $(99 \pm 1 \%$ polarization degree). The integration time at each energy point was set to $0.3 \mathrm{~s}$. Magnetic fields of up to $B=6 \mathrm{~T}$ were applied parallel and antiparallel to the photon beam. The angle of incidence of the beam was varied between $\Theta=0^{\circ}$ (normal incidence) and $\Theta=55^{\circ}$ (oblique incidence) to probe the out-of-plane and in-plane XMCDs. In the 


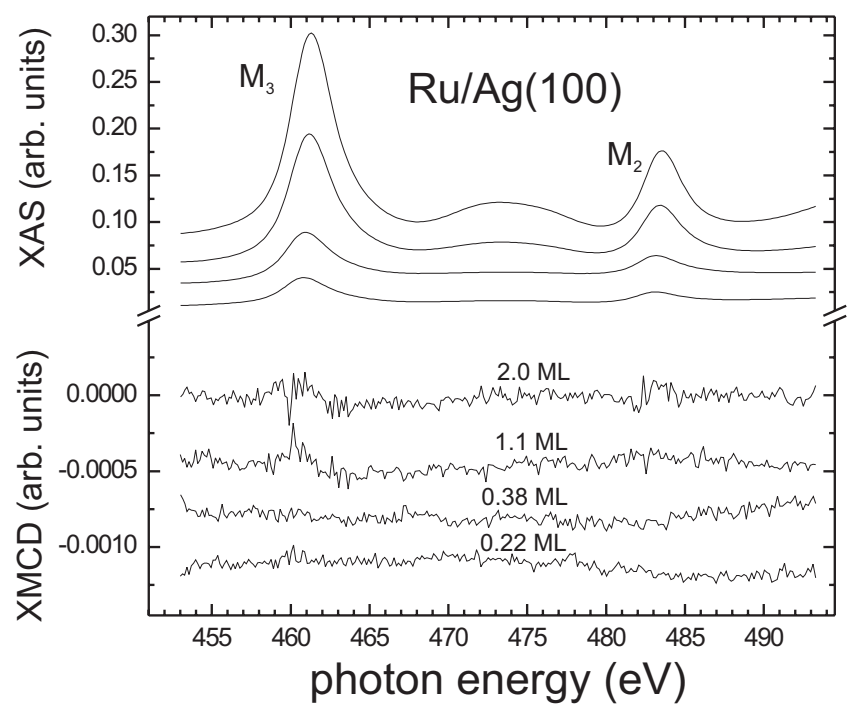

FIG. 2. XAS and XMCD spectra of $\mathrm{Ru}$ on $\mathrm{Ag}(100)$ for coverages $0.22 \pm 0.05,0.38 \pm 0.05,1.1 \pm 0.1$, and $2.0 \pm 0.2 \mathrm{ML}$, measured at $T=5 \mathrm{~K}$ and $B=5 \mathrm{~T}$. The XAS spectra are background subtracted. For clarity, the spectra have been offset in the vertical direction.

following, we refer to the XAS signal as the average intensity $\left(\sigma^{+}+\sigma^{-}\right) / 2$ and to the XMCD as $\left(\sigma^{+}-\sigma^{-}\right)$. The experimental time scales for measuring spectra as well as ramping of the magnets to their designated values are of the order of $10-100 \mathrm{~s}$.

\section{RESULTS}

Figure 2 shows the XAS and XMCD spectra of 0.22-2.0 ML Ru on $\operatorname{Ag}(100)$ recorded at $\Theta=0^{\circ}$. The background XAS of the clean $\mathrm{Ag}(100)$ substrate in the $M_{3,2}$ region (Fig. 1) was subtracted from the original XAS spectra in order to show the residual $\mathrm{Ru}$ signal. The $M_{3}$ and $M_{2}$ edges of Ru stand out clearly, together with a diffuse feature between the two peaks, which is typical of both $\mathrm{Ru}$ and $\mathrm{Rh} .{ }^{21}$ In order to gain magnetic information, the spectra were recorded in an applied field of $B=5 \mathrm{~T}$ at $5 \mathrm{~K}$. In these conditions, even for paramagnetic species, the presence of a magnetic moment on the $4 d$ metal atoms should produce nonzero dichroism with opposite peaks at the $M_{3}$ and $M_{2}$ edges, as observed, e.g., in the case of Fe-induced magnetism in $\mathrm{Ru} / \mathrm{Fe}$ multilayers. ${ }^{21,22}$ The Ru XMCD spectra are instead flat within the noise limit, thus showing that the $\mathrm{Ru}$ magnetic moment is below the sensitivity of our experiment. XMCD spectra at $\Theta=55^{\circ}$, not shown here, give similar results compared to $\Theta=0^{\circ}$ and will not be commented further. Small features appearing in the XMCD around the $M_{3,2}$ edges were proven to be of nonmagnetic origin, as they do not change sign when the direction of the magnetic field is reversed. These artifact features correspond to differences of the order $\pm 0.3 \%$ of the XAS $M_{3}$ edge jump and could be due to the small energy or beam drift during the experiment. According to Tomaz et al., ${ }^{21,22} 1 \mu_{B}$ per $\mathrm{Ru}$ atom produces an XMCD signal of about $8 \%$ with respect to the XAS intensity. If we set our lower detection limit equal to the intensity of the artifact features in the $\mathrm{Ru}$
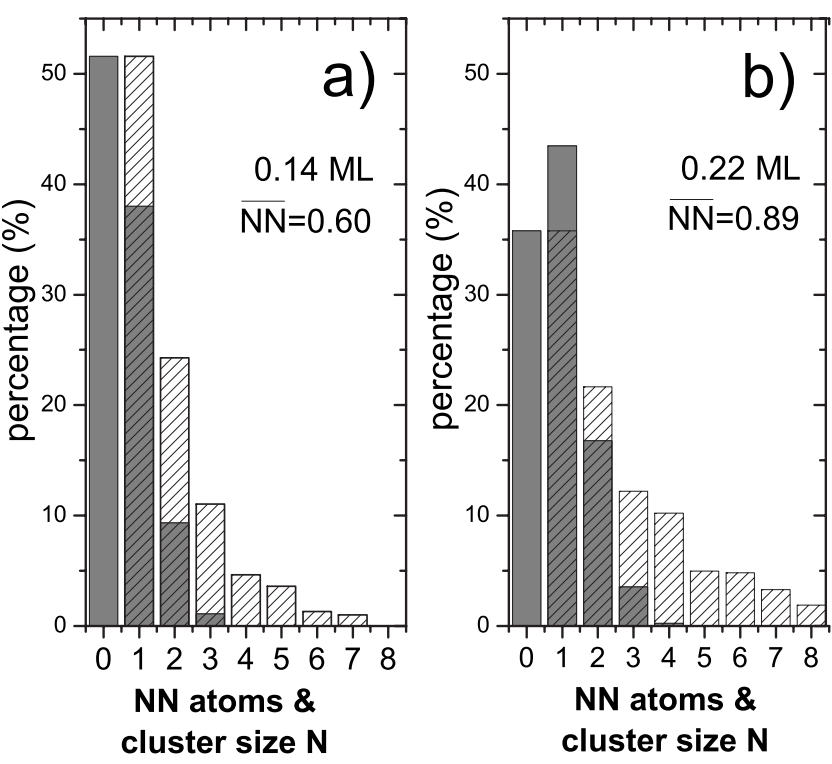

FIG. 3. Histogram of the number of nearest neighbors (NNs) per adatom on a (100) fcc two-dimensional lattice for coverages of (a) 0.14 ML and (b) 0.22 ML assuming random adsorption (gray bars). Hatched bars represent the percentage of atoms included in a given cluster size $N$.

XMCD spectra, we therefore get a sensitivity of about $0.04 \mu_{B}$ per atom. Independently, we can estimate the upper limit of the magnetic moment assuming the validity of the XMCD sum rules as proposed by Carra et al. ${ }^{23}$ From the XMCD noise level of $1 \times 10^{-4}$ a.u. and the integral over the $M_{3,2}$ XAS signal at $0.22 \mathrm{ML}$, we get a value of $0.006 \mu_{B}$ per $\mathrm{Ru}$ atom and hole in the $4 d$ shell. Disregarding charge transfer effects, we get an upper limit of $0.02 \mu_{B}$ per Ru atom. At higher coverages, the upper limit of the moment scales down with the increasing XAS signal.

The measurements in Fig. 2 set a lower bound for the average $\mathrm{Ru}$ magnetic moment at different coverages. However, as mentioned in the Introduction, details in the coordination of the $4 d$ elements are believed to play an important role in the formation of magnetic moments in $4 d$ metals. We therefore need to discuss the growth mode of $\mathrm{Ru}$ and $\mathrm{Rh}$ at low temperature. Deposition of $\mathrm{Rh}$ on a clean $\operatorname{Ag}(100)$ surface at room temperature is known to produce overlayers, which are partially covered by or intermixed with $\mathrm{Ag}$, but which are pseudomorphic with the $\mathrm{Ag}(100)$ substrate. ${ }^{18,24}$ No surface reconstruction has been observed. Also the growth of $\mathrm{Ru}$ on $\mathrm{Ag}(100)$ is expected to be pseudomorphic. ${ }^{11}$ For the samples studied in this work, the low deposition temperature of $5 \mathrm{~K}$ is expected to promote a random pseudomorphic growth with inhibited surface diffusion and, at the same time, to suppress thermally activated intermixing with Ag. Under these conditions, a quantitative estimate of the degree of $4 d-4 d$ coordination at a given coverage can be done using a random occupation model where each pseudomorphic (100) fcc adsorption site is occupied with a probability equal to the coverage in monolayer units. Figure 3 shows the statistics of the number of nearest neighbors (NNs) per adatom for the measured $\mathrm{Ru}$ coverages of 0.14 and $0.22 \mathrm{ML}$ (gray bars). In such a low-coverage regime, funneling events dur- 


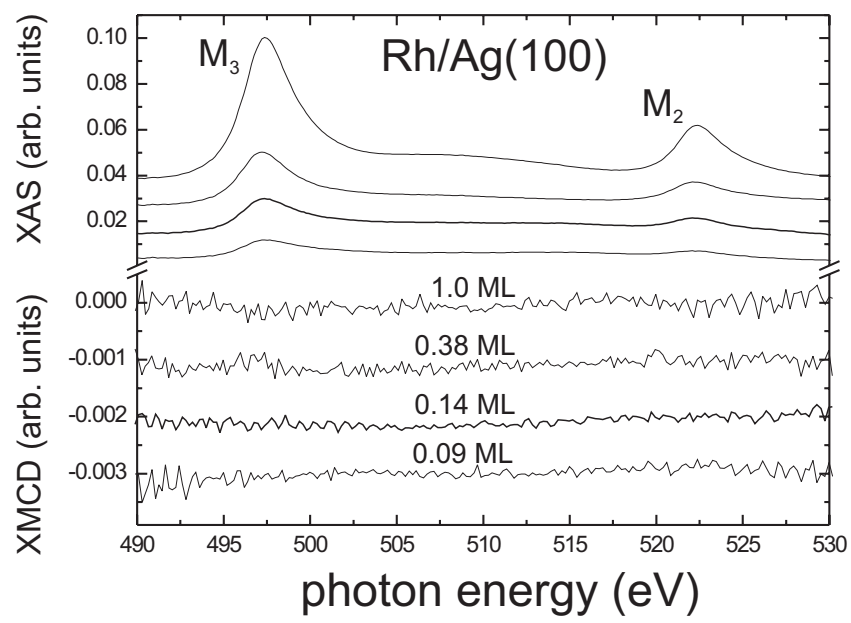

FIG. 4. XAS and XMCD spectra of $\mathrm{Rh}$ on $\mathrm{Ag}(100)$ for coverages of $0.09 \pm 0.05,0.14 \pm 0.05,0.38 \pm 0.1$, and $1.0 \pm 0.1 \mathrm{ML}$ measured at $T=5 \mathrm{~K}$ and $B=6 \mathrm{~T}$. The XAS spectra are background substrated. For clarity, the spectra have been offset in the vertical direction.

ing deposition are expected to be rare, thus providing a rather good estimation of the actual average coordination of $\mathrm{Ru}$ atoms on the surface. In the figure, the NN percentage is given by the gray bars regardless of the actual cluster size $N$, while hatched bars represent the percentage of atoms belonging to clusters of size $N$ (the latter corresponds to $N$ times the percentage of clusters with size $N$ ). We see that the clusters consist mainly of single impurities, dimers, and trimers, and the majority of atoms are included in clusters with size $\leqslant 4$ atoms. Note that a particular cluster size weights in the XAS intensity in proportion to the percentage of the total number of atoms that it contains. Thus, our detection limit of $0.02 \mu_{B}$ per atom has to be renormalized (increased) according to the hatched bars percentages in Fig. 3 in the "worst-case" situation, where only one type of clusters presents a nonzero magnetization. For single impurities in the $0.22 \mathrm{ML} \mathrm{Ru}$ sample, this would mean an upper limit of about $0.06 \mu_{B}$, more than 1 order of magnitude smaller than the predicted $2.2 \mu_{B}$ per atom for single $\mathrm{Ru}$ impurities, ${ }^{3} 1.1-1.6 \mu_{B}$ per $\mathrm{Ru}$ atom in small clusters including dimers and trimers, ${ }^{5}$ and 1.7-1.8 $\mu_{B}$ per $\mathrm{Ru}$ atom in an ideal monolayer on $\mathrm{Ag}(100) .{ }^{13,14}$ We remark that one could in principle deposit a lower amount of material (0.01 ML) to address uniquely single impurities. However, contrary to the $3 d$ elements where XAS-XMCD at the $L_{3,2}$ edges is sensitive to coverages $\leqslant 0.01 \mathrm{ML},{ }^{25,26}$ the lower cross section for $M_{3,2}$ absorption together with the strong substrate background in the energy region of interest limits the XAS sensitivity to about 0.1 ML (Figs. 2, 4, and 5).

Measurements for Rh are shown in Fig. 4 on the $\operatorname{Ag}(100)$ surface and in Fig. 5 on $\mathrm{Pt}(997)$ for coverages between 0.09 and 1.0 ML. All XMCD spectra have been measured at $T$ $=5 \mathrm{~K}$ and $B=6 \mathrm{~T}$. No evidence for the presence of local $\mathrm{Rh}$ magnetic moments is found. ${ }^{27}$ Similar considerations to those discussed for Ru based on the noise level in the XMCD show that the detection limit at the lowest coverage of $\sim 0.1$ ML is about $0.02 \mu_{B}$ per $\mathrm{Rh}$ atom. Theory predicts magnetic

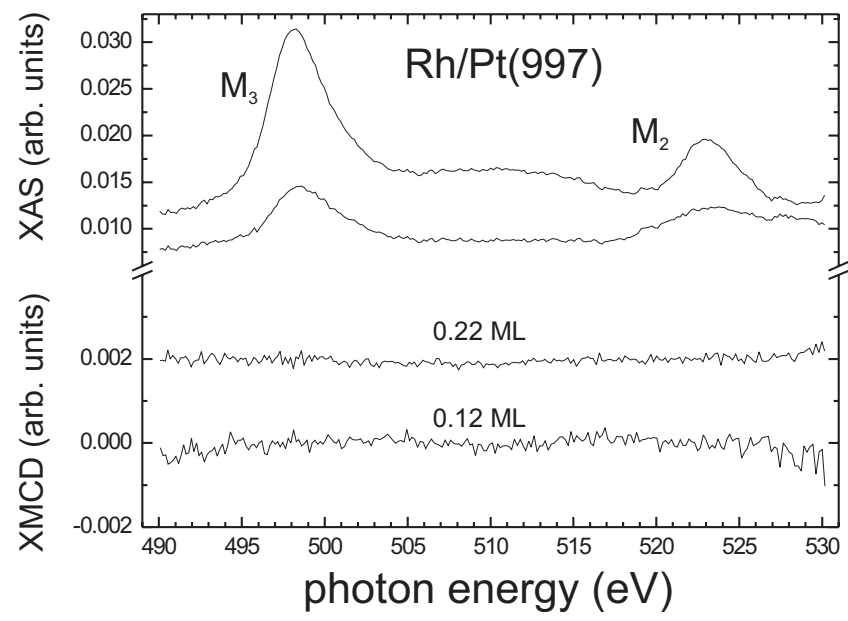

FIG. 5. XAS and XMCD spectra of Rh on $\mathrm{Pt}(997)$ for coverages of $0.12 \pm 0.05$ and $0.22 \pm 0.05$ ML measured at $T=5 \mathrm{~K}$ and $B=5 \mathrm{~T}$. The XAS spectra are background substrated. For clarity, the spectra have been offset in the vertical direction.

moments of $0.3-1.0 \mu_{B}$ per $\mathrm{Rh}$ atom in various cluster configurations with $N=1,2,3,5,9$, and 21 on $\operatorname{Ag}(100),{ }^{4-8}$ where $N$ is the number of $\mathrm{Rh}$ atoms in the cluster. These finite moments are again clearly not observed in our experiment, which should cover cluster sizes with $N=1,2,3,4$, as shown in Fig. 3, and different configurations. The zero XMCD of $\mathrm{Rh}$ on $\mathrm{Pt}(997)$, however, was expected on the basis of $a b$ initio calculations of a nonmagnetic state for $\mathrm{Rh}$ impurities on $\mathrm{Pt}(100){ }^{28}$ This is a general trend of $5 d$ vs $4 d$ substrates since, compared to $\mathrm{Ag}$, the larger extension of the Pt $5 d$ wave functions favors the hybridization between adatoms and surface and reduces the impurity magnetic moment. $^{28}$

\section{DISCUSSION}

We discuss here the possible origin of the discrepancy between a large number of consistent theoretical results and the missing local moments in $\mathrm{Ru}$ and $\mathrm{Rh}$ observed by XMCD.

Alloying of $4 d$ metals with the substrate. This is a widely accepted argument for interpreting the absence of ferromagnetism in Rh monolayers probed by MOKE. ${ }^{15,16}$ STM, Auger, thermal desorption, and ion scattering studies show that ideal layer-by-layer growth of $\mathrm{Rh}$ and $\mathrm{Ru}$ on noble metal surfaces can hardly be achieved at room temperature, due to the elemental difference of surface free energy that promotes Rh-Ag interdiffusion. ${ }^{24,29}$ Turek et al. calculated a substantial decrease of both the $\mathrm{Ru}$ and $\mathrm{Rh}$ magnetic moments in subsurface positions for mixed $\mathrm{Ru}, \mathrm{Rh} / \mathrm{Ag}(100)$ layers. ${ }^{14}$ For small clusters, on the other hand, Stepanyuk et al. found an increase of the $\mathrm{Ru}$ and $\mathrm{Rh}$ moment upon mixing with Ag. ${ }^{5} \mathrm{In}$ our samples, we expect alloying to be suppressed due to deposition at $5 \mathrm{~K}$. Even in the case of alloying and reduced magnetization, however, the $\mathrm{Ru}$ and $\mathrm{Rh}$ moments should be detectable as they are expected to be larger than our error margins. 
Relaxation effects in band-structure calculations. Most $a b$ initio calculations of the $4 d$ systems have been carried out without fully relaxing the atomic lattice of the overlayer and the substrate, often by assuming bulk-truncated pseudomorphic configurations. When relaxation has been taken into account, this was done by taking fixed average interlayer spacings. ${ }^{14} \mathrm{Wu}$ and Freeman noted that relaxation shall not affect the tendency of $4 d$ atoms to couple ferromagnetically. ${ }^{11}$ However, due to the overlap of the electron wave functions, small differences in the interatomic distance between the $4 d$ atoms and the substrate, or between $4 d$ atoms in a cluster, ${ }^{30}$ could induce major changes in the magnitude of the local magnetic moments. Such effects have not been specifically addressed in theoretical treatments, perhaps for the lack of related experimental information. Relaxing the $\mathrm{Ru}$ and $\mathrm{Rh}$ position toward the substrate might partially or totally quench the local $4 d$ moment.

Many-body effects. Phenomena such as the Kondo effect or local spin fluctuations might effectively reduce the magnetic moment of impurities in metal hosts. While these effects have to be considered in experiments carried out at finite temperature, they are normally not included in ab initio density functional treatments. For a fluctuating valence system with magnetic moment averaging to zero, XMCD measurements are clearly too slow to provide magnetic information. In such a case, the motion of the magnetic moment would no longer be dominated by the combined action of thermal fluctuations and applied magnetic field but by intrinsic fluctuations due to interactions with the conduction electrons. The magnetoresistance measurements by Beckmann and Bergmann seem to support this hypothesis, showing that the dephasing rate of electrons scattered off $\mathrm{Ru}$ and $\mathrm{Rh}$ impurities is suppressed at low temperature as a result of screening of the local $4 d$ moments. ${ }^{19}$ As already noted by these authors, however, Kondo screening and spin fluctuations are expected to become less important for large clusters and therefore cannot explain the missing magnetic moments in the whole coverage range of the present investigation.

It is possible that more than one effect concurs to determine the nonmagnetic state of $\mathrm{Ru}$ and $\mathrm{Rh}$ atoms in impurities and clusters revealed by XMCD. With respect to structural relaxation, experimental methods usually do not allow to determine the interatomic distance between isolated impurities and the substrate. However, detailed $a b$ initio calculations could address this point. Mössbauer spectroscopy on ${ }^{99} \mathrm{Ru}$ isotopes could provide information on the coordination state of Ru. STM spectroscopy performed on individual impurities and clusters, on the other hand, might reveal the role of Kondo screening in these systems. ${ }^{31}$

\section{CONCLUSIONS}

We have performed element-specific measurements of the local magnetic moment of the $4 d$ metals $\mathrm{Ru}$ and Rh deposited at $5 \mathrm{~K}$ on $\mathrm{Ag}(100)$ and $\operatorname{Pt}(997)$. Ru was investigated in the coverage range from 0.22 to $2.0 \mathrm{ML}$ on $\mathrm{Ag}(100)$. $\mathrm{Rh}$ was probed in the 0.09-1.0 ML coverage range on $\mathrm{Ag}(100)$ and $\mathrm{Pt}(997)$ surfaces. No magnetic moments were detected in both $\mathrm{Ru}$ and $\mathrm{Rh}$ impurities, clusters, and quench-condensed layers within the detection limit of $0.02 \mu_{B}$ per atom and in the presence of applied magnetic fields of up to $6 \mathrm{~T}$. These results show that the lack of ferromagnetic order in Rh films observed by MOKE can be attributed to the quenching of the $4 d$ atomic moment upon deposition on nonmagnetic noble metal substrates, even without taking into account imperfect monolayer growth. The XMCD data are in contrast with $a b$ initio density functional calculations: while $\mathrm{Rh}$ impurities on $\mathrm{Ag}(100)$ represent a borderline magnetic-nonmagnetic system in different theoretical models, ${ }^{3-6} \mathrm{Rh}$ clusters containing a few atoms, Ru impurities, clusters, and monolayers are predicted to have sizable magnetic moments of the order of $1 \mu_{B}$ per atom, ${ }^{3-6,8-14}$ which are clearly not observed in the present experiment. The magnetic behavior of $4 d$ elements deposited on nonmagnetic metal surfaces appears to be a complex problem that cannot be entirely treated in the framework of relaxation-free, zero temperature density functional models. On the experimental side, the possible role of local spin fluctuations as a function of temperature and cluster size remains to be clarified.

\section{ACKNOWLEDGMENTS}

The authors acknowledge the European Synchrotron Radiation Facility for provision of beam time and thank Gilles Retout for technical assistance in using beamline ID08.

\footnotetext{
*j.honolka@fkf.mpg.de

${ }^{1}$ A. J. Cox, J. G. Louderback, and L. A. Bloomfield, Phys. Rev. Lett. 71, 923 (1993).

${ }^{2}$ A. J. Cox, J. G. Louderback, S. E. Apsel, and L. A. Bloomfield, Phys. Rev. B 49, 12295 (1994).

${ }^{3}$ P. Lang, V. S. Stepanyuk, K. Wildberger, R. Zeller, and P. H. Dederichs, Solid State Commun. 92, 755 (1994).

${ }^{4}$ K. Wildberger, V. S. Stepanyuk, P. Lang, R. Zeller, and P. H. Dederichs, Phys. Rev. Lett. 75, 509 (1995).

${ }^{5}$ V. S. Stepanyuk, W. Hergert, P. Rennert, K. Wildberger, R. Zeller, and P. H. Dederichs, Phys. Rev. B 59, 1681 (1999).

${ }^{6}$ I. Cabria, B. Nonas, R. Zeller, and P. H. Dederichs, Phys. Rev. B
}

65, 054414 (2002).

${ }^{7}$ D. I. Bazhanov, W. Hergert, V. S. Stepanyuk, A. A. Katsnelson, P. Rennert, K. Kokko, and C. Demangeat, Phys. Rev. B 62, 6415 (2000).

${ }^{8}$ V. Bellini, N. Papanikolaou, R. Zeller, and P. H. Dederichs, Phys. Rev. B 64, 094403 (2001).

${ }^{9}$ O. Eriksson, R. C. Albers, and A. M. Boring, Phys. Rev. Lett. 66, 1350 (1991).

${ }^{10}$ M. J. Zhu, D. M. Bylander, and L. Kleinman, Phys. Rev. B 43, 4007 (1991).

${ }^{11}$ R. Wu and A. J. Freeman, Phys. Rev. B 45, 7222 (1992).

${ }^{12}$ S. Blügel, Phys. Rev. Lett. 68, 851 (1992). 
${ }^{13}$ S. Blügel, Phys. Rev. B 51, 2025 (1995).

${ }^{14}$ I. Turek, J. Kudrnovský, M. Šob, V. Drchal, and P. Weinberger, Phys. Rev. Lett. 74, 2551 (1995).

${ }^{15}$ G. A. Mulhollan, R. L. Fink, and J. L. Erskine, Phys. Rev. B 44, 2393 (1991).

${ }^{16}$ C. Liu and S. D. Bader, Phys. Rev. B 44, 12062 (1991).

${ }^{17}$ I. Chado, F. Scheurer, and J. P. Bucher, Phys. Rev. B 64, 094410 (2001).

${ }^{18}$ H. Li, S. C. Wu, D. Tian, Y. S. Li, J. Quinn, and F. Jona, Phys. Rev. B 44, 1438 (1991).

${ }^{19}$ H. Beckmann and G. Bergmann, Phys. Rev. B 55, 14350 (1997).

${ }^{20}$ G. Bergmann, Phys. Rev. B 28, 2914 (1983).

${ }^{21}$ M. A. Tomaz, T. Lin, G. R. Harp, E. Hallin, T. K. Sham, and W. L. O’Brien, J. Vac. Sci. Technol. A 16, 1359 (1998).

${ }^{22}$ T. Lin, M. A. Tomaz, M. M. Schwickert, and G. R. Harp, Phys. Rev. B 58, 862 (1998).

${ }^{23}$ P. Carra, B. T. Thole, Massimo Altarelli, and Xingdong Wang, Phys. Rev. Lett. 70, 694 (1993).

${ }^{24}$ S.-L. Chang, J.-M. Wen, P. A. Thiel, S. Günther, J. A. Meyer, and
R. J. Behm, Phys. Rev. B 53, 13747 (1996).

${ }^{25}$ P. Gambardella, S. S. Dhesi, S. Gardonio, C. Grazioli, P. Ohresser, and C. Carbone, Phys. Rev. Lett. 88, 047202 (2002).

${ }^{26}$ P. Gambardella, S. Rusponi, M. Veronese, S. S. Dhesi, C. Grazioli, A. Dallmeyer, I. Cabria, R. Zeller, P. H. Dederichs, K. Kern, C. Carbone, and H. Brune, Science 300, 1130 (2003).

${ }^{27}$ A calibration of the Rh $M_{3,2}$ XMCD signal vs magnetic moment is given in, e.g., M. A. Tomaz, D. C. Ingram, G. R. Harp, D. Lederman, E. Mayo, and W. L. O’Brien, Phys. Rev. B 56, 5474 (1997).

${ }^{28}$ V. S. Stepanyuk, W. Hergert, K. Wildberger, R. Zeller, and P. H. Dederichs, Phys. Rev. B 53, 2121 (1996).

${ }^{29}$ P. J. Schmitz, W.-Y. Leung, G. W. Graham, and P. A. Thiel, Phys. Rev. B 40, 11477 (1989).

${ }^{30}$ Y. Mokrousov, G. Bihlmayer, S. Heinze, and S. Blügel, Phys. Rev. Lett. 96, 147201 (2006).

${ }^{31}$ P. Wahl, L. Diekhöner, M. A. Schneider, L. Vitali, G. Wittich, and K. Kern, Phys. Rev. Lett. 93, 176603 (2004). 